\title{
Construction of a New Class of Bent and Semi-bent Functions
}

\author{
P. L. Sharma \\ Department of Mathematics \\ Himachal Pradesh University, \\ Shimla 171005
}

\author{
Neetu Dhiman \\ Department of Mathematics \\ Himachal Pradesh University, \\ Shimla 171005
}

\begin{abstract}
Bent functions play an important role in the designing of Sboxes. These functions also have significant applications in coding theory, graph theory and sequence design. In the literature of bent functions their complete classification and characterization is still elusive, so the constructions and characterizations of bent functions are challenging problems. Many constructions methods and characterizations of bent functions are discussed in the literature. In this paper we obtain a new infinite class of bent and semi-bent functions using few Walsh transform values.
\end{abstract}

\section{Keywords}

Boolean functions, Walsh-Hadamard transform, Bent functions, Semi-bent functions.

\section{INTRODUCTION}

Rothaus [1] had introduced the bent functions in 1976. Due to the highest non-linearity of bent functions, they have gained importance in the designing of stream ciphers and block ciphers. Kumar et al. [2] extended Rothaus's definition of bent functions to generalized bent functions and also discussed their properties. Since 1974, bent functions are extensively studied because of their significant applications in cryptography (in the design of stream ciphers and in the substitution boxes of block ciphers) [3], coding theory [4], sequence design [5] and graph theory [6,7]. The new structure introduced in the literature of mathematics known as Rhotrix is gaining importance for making the cryptosystems more secure, see $[8,9,10,11]$. Irreducible polynomial play an important role in the structure of finite fields which is an essential tool in cryptography, see [12]. Bent functions are not balanced. A complete classification and characterization of bent functions is still elusive, so the construction and characterization of bent functions are challenging problems. In the recent time most of the research work have been done on the construction of bent functions. Primary and secondary constructions of bent functions are the two kinds of construction of bent functions. In the primary construction, there is no use of previously existing bent functions to construct new ones, while in secondary construction some previously known bent functions are used to construct new bent functions, see $[13,14,15,16]$. Several constructions of bent functions are discussed in $[17,18]$. Some constructions and characterizations of gbent functions are discussed in $[19,20]$.

Some new constructions of bent and semi-bent functions are recently introduced by $\mathrm{Xu}$ et al. [21]. We here present a new construction of bent functions.

Any function $f(x): F_{2^{n}} \rightarrow F_{2}$ is called a Boolean function. Let $n=2 m$ be a positive integer and $F_{2^{n}}$ be the finite field with $2^{\mathrm{n}}$ elements. Let $F_{2^{n}}^{*}=F_{2^{n}} \backslash\{0\}$. For any positive integer $n$, and $r$ dividing $n$, the trace function from $F_{2^{n}} \rightarrow F_{2^{r}}$ denoted by $\operatorname{Tr}_{r}^{n}(x)$, is the mapping defined for every $x \in F_{2^{n}}$ as:

$\operatorname{Tr}_{r}^{n}(x)=\sum_{i=0}^{\frac{n}{r}-1} x^{2^{i r}}=x+x^{2^{r}}+x^{2^{2 r}}+\cdots+x^{2^{n-r}}$.

In particular, the absolute trace occurs for $r=1$. In deriving our results we use some known properties of the trace function such as $\operatorname{Tr}_{1}^{n}(x)=\operatorname{Tr}_{1}^{n}\left(x^{2}\right)$ and for every integer $r$ dividing $n$, the transitivity property of $\operatorname{Tr}_{r}^{n}(x)$, that is $\operatorname{Tr}_{1}^{n}(x)=\operatorname{Tr}_{1}^{r}(x) \circ \operatorname{Tr}_{r}^{n}(x)$. The Walsh-Hadamard transform of a Boolean function $f(x): F_{2^{n}} \rightarrow F_{2}$ is the function $\widehat{\chi_{f}}: F_{2^{n}} \rightarrow Z$ defined by

$\widehat{\chi_{f}}(w)=\sum_{x \in F_{2^{n}}}(-1)^{f(x)+\operatorname{Tr}_{1}^{n}(w x)}$, for all $w \in F_{2^{n}}$.

The values $\widehat{\chi_{f}}(w)$, for all $w \in F_{2^{n}}$ are called the Walsh coefficients of $f$ and the multiset $\left\{\widehat{\chi_{f}}(w), w \in F_{2^{n}}\right\}$ is called the Walsh spectrum of a Boolean function $f$. If $n$ is even, a Boolean function $f: F_{2^{n}} \rightarrow F_{2}$ is said to be bent if $\widehat{\chi_{f}}(w)=$ $\pm 2^{\frac{n}{2}}$, for all $w \in F_{2^{n}}$ and $f$ is said to be semi-bent if $\widehat{\chi_{f}}(w)=\left\{0, \pm 2^{\frac{n}{2}+1}\right\}$ for all $w \in F_{2^{n}}$.

\section{MAIN RESULTS}

We discuss the Walsh-Hadamard transform of a Boolean function $f(x)$ in the following Lemma.

Lemma 2.1 Let $n$ be a positive integer and $a, b, c \in F_{2^{n}}^{*}$. Let $g(x)$ be a Boolean function over $F_{2^{n}}$. Define the Boolean function $f(x)$ by

$f(x)=g(x)+\operatorname{Tr}_{1}^{n}(a x) \operatorname{Tr}_{1}^{n}(b x)+\operatorname{Tr}_{1}^{n}(a x) \operatorname{Tr}_{1}^{n}(c x)$,

then for all $w \in F_{2^{n}}$

$\widehat{\chi_{f}}(w)=\frac{1}{2}\left[\widehat{\chi_{g}}(w)+\widehat{\chi_{g}}(w+a)+\widehat{\chi_{g}}(w+b+c)-\right.$ $\left.\widehat{\chi_{g}}(w+a+b+c)\right]$.

Proof. For $i, j \in\{0,1\}$ and $a, b \in F_{2^{n}}^{*}$, define

$T_{(i, j)}=\left\{x \in F_{2^{n}}: \operatorname{Tr}_{1}^{n}(a x)=i, \operatorname{Tr}_{1}^{n}(b x)=j\right\}$

and denote

$S_{(i, j)}(w)=\sum_{x \in T_{(i, j)}} \omega^{g(x)+T r_{1}^{n}(w x)}$

and

$Q_{(i, j)}(w+c)=\sum_{x \in T_{(i, j)}} \omega^{g(x)+T r_{1}^{n}((w+c) x)}$.

For each $w \in F_{2^{n}}$, we have

$$
\begin{aligned}
\widehat{\chi_{f}}(w) & =\sum_{x \in F_{2^{n}}}(-1)^{f(x)+T r_{1}^{n}(w x)} \\
= & \sum_{x \in F_{2^{n}}}(-1)^{g(x)+T r_{1}^{n}(a x) T r_{1}^{n}(b x)+T r_{1}^{n}(a x) T r_{1}^{n}(c x)+T r_{1}^{n}(w x)}
\end{aligned}
$$


$=\sum_{x \in T_{(0,0)}}(-1)^{g(x)+\operatorname{Tr}_{1}^{n}(w x)}+\sum_{x \in T_{(0,1)}}(-1)^{g(x)+\operatorname{Tr}_{1}^{n}(w x)}$

$+\sum_{x \in T_{(1,0)}}(-1)^{g(x)+\operatorname{Tr}_{1}^{n}(c x)+\operatorname{Tr}_{1}^{n}(w x)}$

$+\sum_{x \in T_{(1,1)}}(-1)^{g(x)+\operatorname{Tr}_{1}^{n}(c x)+\operatorname{Tr}_{1}^{n}(w x)+1}$

$=S_{(0,0)}(w)+S_{(0,1)}(w)+Q_{(1,0)}(w+c)-Q_{(1,1)}(w+c)$.

Now

$$
\begin{aligned}
\widehat{\chi_{g}} & (w)=\sum_{x \in F_{2^{n}}}(-1)^{g(x)+\operatorname{Tr}_{1}^{n}(w x)} \\
& =\sum_{x \in T_{(0,0)}}(-1)^{g(x)+\operatorname{Tr}_{1}^{n}(w x)}+\sum_{x \in T_{(0,1)}}(-1)^{g(x)+\operatorname{Tr}_{1}^{n}(w x)} \\
& +\sum_{x \in T_{(1,0)}}(-1)^{g(x)+\operatorname{Tr}_{1}^{n}(w x)}+\sum_{x \in T_{(1,1)}}(-1)^{g(x)+\operatorname{Tr}_{1}^{n}(w x)} \\
& =S_{(0,0)}(w)+S_{(0,1)}(w)+S_{(1,0)}(w)+S_{(1,1)}(w) .
\end{aligned}
$$

Therefore,

$S_{(0,0)}(w)+S_{(0,1)}(w)=\widehat{\chi_{g}}(w)-S_{(1,0)}(w)-S_{(1,1)}(w)$.

Using (2.6) in (2.5), we get

$$
\begin{gathered}
\widehat{\chi_{f}}(w)=\widehat{\chi_{g}}(w)-S_{(1,0)}(w)-S_{(1,1)}(w)+Q_{(1,0)}(w+c)- \\
Q_{(1,1)}(w+c) .
\end{gathered}
$$

To compute, $S_{(1,0)}(w)$ and $S_{(1,1)}(w)$ solving the following system

$$
\left[\begin{array}{cccc}
1 & 1 & 1 & 1 \\
1 & -1 & -1 & -1 \\
1 & 1 & -1 & -1 \\
1 & -1 & -1 & 1
\end{array}\right]\left[\begin{array}{l}
\mathbf{S}_{(0,0)}(w) \\
\mathbf{S}_{(1,0)}(w) \\
\mathbf{S}_{(0,1)}(w) \\
\mathbf{S}_{(1,1)}(w)
\end{array}\right]=\left[\begin{array}{c}
\tilde{\chi}_{g}^{(w)} \\
\tilde{\chi}_{g}^{(w+b)} \\
\tilde{\chi}_{g}^{(w+a)} \\
\tilde{\chi}_{g}^{(w+a+b)}
\end{array}\right]
$$

that is

$S_{(0,0)}(w)+S_{(1,0)}(w)+S_{(0,1)}(w)+S_{(1,1)}(w)=\widetilde{\chi_{g}}(w)$,

$S_{(0,0)}(w)-S_{(1,0)}(w)+S_{(0,1)}(w)-S_{(1,1)}(w)=\widetilde{\chi_{g}}(w+b)$,

$S_{(0,0)}(w)+S_{(1,0)}(w)-S_{(0,1)}(w)-S_{(1,1)}(w)=\widetilde{\chi_{g}}(w+a)$,

$$
\begin{array}{r}
S_{(0,0)}(w)-S_{(1,0)}(w)-S_{(0,1)}(w)+S_{(1,1)}(w)= \\
\widetilde{\chi_{g}}(w+a+b) .
\end{array}
$$

Solving equations from (2.8) - (2.11), we get

$$
\begin{gathered}
S_{(1,0)}(w)=\frac{1}{4}\left\{\widehat{\chi_{g}}(w)+\widehat{\chi_{g}}(w+b)-\widehat{\chi_{g}}(w+a)-\right. \\
\left.\widehat{\chi_{g}}(w+a+b)\right\}
\end{gathered}
$$

and

$$
\begin{gathered}
S_{(1,1)}(w)=\frac{1}{4}\left\{\widehat{\chi_{g}}(w)-\widehat{\chi_{g}}(w+b)-\widehat{\chi_{g}}(w+a)+\right. \\
\left.\widehat{\chi_{g}}(w+a+b)\right\} .
\end{gathered}
$$

Substituting $w=w+c$ in (2.12) and (2.13), we get

$$
\begin{aligned}
Q_{(1,0)}(w+c)= & \frac{1}{4}\left\{\widehat{\chi_{g}}(w+c)+\widehat{\chi_{g}}(w+b+c)-\right. \\
& \left.\widehat{\chi_{g}}(w+a+c)-\widehat{\chi_{g}}(w+a+b+c)\right\}
\end{aligned}
$$

and

$$
\begin{aligned}
Q_{(1,1)}(w+c) & =\frac{1}{4}\left\{\widehat{\chi_{g}}(w+c)-\widehat{\chi_{g}}(w+b+c)-\right. \\
& \left.\widehat{\chi_{g}}(w+a+c)+\widehat{\chi_{g}}(w+a+b+c)\right\} .
\end{aligned}
$$

Using (2.12) - (2.15) in (2.7), we get

$\widehat{\chi_{f}}(w)=\widehat{\chi_{g}}(w)-\frac{1}{4}\left\{\widehat{\chi_{g}}(w)+\widehat{\chi_{g}}(w+b)-\widehat{\chi_{g}}(w+a)-\right.$ $\left.\widehat{\chi_{g}}(w+a+b)\right\}-\frac{1}{4}\left\{\widehat{\chi_{g}}(w)-\widehat{\chi_{g}}(w+b)-\widehat{\chi_{g}}(w+a)+\right.$ $\left.\widehat{\chi_{g}}(w+a+b)\right\}+\frac{1}{4}\left\{\widehat{\chi_{g}}(w+c)+\widehat{\chi_{g}}(w+b+c)-\right.$ $\left.\widehat{\chi_{g}}(w+a+c)-\widehat{\chi_{g}}(w+a+b+c)\right\}-\frac{1}{4}\left\{\widehat{\chi_{g}}(w+c)-\right.$ $\left.\widehat{\chi_{g}}(w+b+c)-\widehat{\chi_{g}}(w+a+c)+\widehat{\chi_{g}}(w+a+b+c)\right\}$ $=\frac{1}{4}\left\{4 \widehat{\chi_{g}}(w)-\widehat{\chi_{g}}(w)-\widehat{\chi_{g}}(w+b)+\widehat{\chi_{g}}(w+a)+\right.$ $\widehat{\chi_{g}}(w+a+b)-\widehat{\chi_{g}}(w)+\widehat{\chi_{g}}(w+b)-\widehat{\chi_{g}}(w+a+b)+$ $\widehat{\chi_{g}}(w+a)+\widehat{\chi_{g}}(w+c)+\widehat{\chi_{g}}(w+b+c)-\widehat{\chi_{g}}(w+a+$ $c)-\widehat{\chi_{g}}(w+a+b+c)-\widehat{\chi_{g}}(w+c)+\widehat{\chi_{g}}(w+b+c)+$ $\left.\widehat{\chi_{g}}(w+a+c)-\widehat{\chi_{g}}(w+a+b+c)\right\}$

$$
\begin{aligned}
= & \frac{1}{4}\left\{2 \widehat{\chi_{g}}(w)+2 \widehat{\chi_{g}}(w+a)+2 \widehat{\chi_{g}}(w+b+c)-\right. \\
& \left.2 \widehat{\chi_{g}}(w+a+b+c)\right\} \\
= & \frac{1}{2}\left\{\widehat{\chi_{g}}(w)+\widehat{\chi_{g}}(w+a)+\widehat{\chi_{g}}(w+b+c)-\widehat{\chi_{g}}(w+a+\right. \\
& \quad b+c)\} .
\end{aligned}
$$

Theorem 2.2 Let $\boldsymbol{k}$ be a positive integer such that $\boldsymbol{k}>1$ and let $\boldsymbol{a}, \boldsymbol{b}, \boldsymbol{c}$ be three distinct elements in $\boldsymbol{F}_{2^{4 k}}^{*}$ such that $\boldsymbol{a}+\boldsymbol{b}+$ $c \neq \mathbf{0}$. Let $\lambda \in \boldsymbol{F}_{2^{4 k}}^{*}$ such that $\lambda+\lambda^{2^{3 k}}=\mathbf{1}$. Define the Boolean function $\boldsymbol{f}(\boldsymbol{x})$ as

$f(x)=$

$\operatorname{Tr}_{1}^{4 k}\left(\lambda x^{2 k+1}\right)+\operatorname{Tr}_{1}^{4 k}(a x) T r_{1}^{4 k}(b x)+\operatorname{Tr}_{1}^{4 k}(a x) T r_{1}^{4 k}(c x)$.

Then the function $\boldsymbol{f}(\boldsymbol{x})$ is bent if

$\operatorname{Tr}_{1}^{4 k}\left(\lambda\left(a^{2^{k}} b+b^{2^{k}} a\right)\right)=\operatorname{Tr}_{1}^{4 k}\left(\lambda\left(b^{2^{k}} c+c^{2^{k}} b\right)\right)=$ $\operatorname{Tr}_{1}^{4 k}\left(\lambda\left(a^{2^{k}} c+c^{2^{k}} a\right)\right)=0$

and $f(x)$ is semi-bent if any one of the $\operatorname{Tr}_{1}^{4 k}\left(\lambda\left(a^{2^{k}} b+\right.\right.$ $\left.\left.b^{2^{k}} a\right)\right), \operatorname{Tr}_{1}^{4 k}\left(\lambda\left(b^{2^{k}} c+c^{2^{k}} b\right)\right)$ and $\operatorname{Tr}_{1}^{4 k}\left(\lambda\left(a^{2^{k}} c+\right.\right.$ $\left.\left.c^{2^{k}} a\right)\right)$ is 1 and the other two are zero.

\section{Proof. Let}

$g(x)=\operatorname{Tr}_{1}^{4 k}\left(\lambda x^{2 k+1}\right)$.

For each $w \in F_{2^{n}}$, we have from Lemma 2.1

$$
\begin{aligned}
\widehat{\chi_{f}}(w)= & \frac{1}{2}\left\{\widehat{\chi_{g}}(w)+\widehat{\chi_{g}}(w+a)+\widehat{\chi_{g}}(w+b+c)-\right. \\
& \left.\widehat{\chi_{g}}(w+a+b+c)\right\} \\
= & \Delta_{1}+\Delta_{2},
\end{aligned}
$$

where

$\Delta_{1}=\frac{1}{2}\left\{\widehat{\chi_{g}}(w)+\widehat{\chi_{g}}(w+a)\right\}$

and 
$\Delta_{2}=\frac{1}{2}\left\{\widehat{\chi_{g}}(w+b+c)-\widehat{\chi_{g}}(w+a+b+c)\right\}$.

Using

$\widehat{\chi_{g}}(w)=2^{2 k}(-1)^{T r_{1}^{4 k}\left(\lambda w^{2^{k}+1}\right)}$

to find the values of $\Delta_{1}$ and $\Delta_{2}$. Therefore,

$$
\begin{aligned}
& \Delta_{1}=\frac{1}{2} 2^{2 k}\left\{(-1)^{T r_{1}^{4 k}\left(\lambda w^{2^{k}+1}\right)}+(-1)^{T r_{1}^{4 k}\left(\lambda(w+a)^{2^{k}+1}\right)}\right\} \\
&= \frac{1}{2} 2^{2 k}\left\{(-1)^{T r_{1}^{4 k}\left(\lambda w^{2^{k}+1}\right)}+\right. \\
&(-1)^{\left.T r_{1}^{4 k}\left(\lambda\left(w^{2^{k}} a+w a^{2^{k}}+w^{2^{k}+1}+a^{2^{k}+1}\right)\right)\right\}} \\
&= \frac{1}{2} 2^{2 k}(-1)^{\operatorname{Tr}_{1}^{4 k}\left(\lambda w^{2^{k}+1}\right)}\left\{1+(-1)^{T r_{1}^{4 k}\left(\lambda\left(w^{2^{k}} a+w a^{2^{k}}+a^{2^{k}+1}\right)\right)}\right\}
\end{aligned}
$$

and

$$
\begin{aligned}
& \Delta_{2}= \\
& \frac{1}{2} 2^{2 k}\left\{(-1)^{T r_{1}^{4}\left(\lambda(w+b+c)^{2^{k}+1}\right)}-(-1)^{T r_{1}^{4}\left(\lambda(w+a+b+c)^{2^{k}+1}\right)}\right\}
\end{aligned}
$$

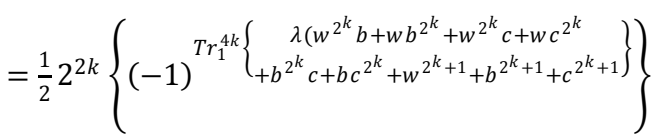

$$
\begin{aligned}
& -\frac{1}{2} 2^{2 k}\left\{(-1) \quad \operatorname{Tr}_{1}^{4 k}\left\{\begin{array}{c}
\lambda\left(w^{2^{k}} a+w a^{2^{k}}+w^{2^{k}} b+w b^{2^{k}}\right. \\
+w^{2^{k}} c+w c^{2^{k}}+a^{2^{k}} b+a b^{2^{k}} \\
+a^{2^{k}} c+a c^{2^{k}}+b^{2^{k}} c+b c^{2^{k}} \\
+w^{2^{k}+1}+a^{2^{k}+1}+b^{2^{k}+1}+c^{2^{k}+1}
\end{array}\right\}\right. \text {. }
\end{aligned}
$$

Let

$c_{1}=\operatorname{Tr}_{1}^{4 k}\left\{\lambda\left(\left(w^{2^{k}} a+w a^{2^{k}}+a^{2^{k}+1}\right)\right\}\right.$,

$c_{2}=\operatorname{Tr}_{1}^{4 k}\left\{\lambda\left(w^{2^{k}} b+w b^{2^{k}}+w^{2^{k}} c+w c^{2^{k}}+b^{2^{k}+1}+\right.\right.$ $\left.c^{2^{k}+1}\right\}$,

$t_{1}=\operatorname{Tr}_{1}^{4 k}\left\{\lambda\left(a^{2^{k}} b+a b^{2^{k}}\right)\right\}$

$t_{2}=\operatorname{Tr}_{1}^{4 k}\left\{\lambda\left(b^{2^{k}} c+b c^{2^{k}}\right)\right\}$,

and

$t_{3}=\operatorname{Tr}_{1}^{4 k}\left\{\lambda\left(a^{2^{k}} c+a c^{2^{k}}\right)\right\}$.

Using (2.19) - (2.23) in (2.17) and (2.18), we have

$\Delta_{1}=\frac{1}{2} 2^{2 k}(-1)^{T r_{1}^{4 k}\left(\lambda w^{2^{k}+1}\right)}\left\{1+(-1)^{c_{1}}\right\}$

and
$\Delta_{2}=$

$\frac{1}{2} 2^{2 k}(-1)^{T r_{1}^{4 k}\left(\lambda w^{2^{k}+1}\right)+c_{2}}\left\{(-1)^{t_{2}}-\right.$

$\left.(-1)^{c_{1}+t_{1}+t_{2}+t_{3}}\right\}$.

For $t_{1}=t_{2}=t_{3}=0$, we have

$\Delta_{2}=\frac{1}{2} 2^{2 k}(-1)^{T r_{1}^{4 k}\left(\lambda w^{2^{k}+1}\right)+c_{2}}\left\{1-(-1)^{c_{1}}\right\}$.

If $c_{2}=0$, then from (2.24) and (2.26), we have

$\widehat{\chi_{f}}(w)=\Delta_{1}+\Delta_{2}=\frac{1}{2} 2^{2 k}(-1)^{T r_{1}^{4 k}\left(\lambda w^{2^{k}+1}\right)}\left\{1+(-1)^{c_{1}}+\right.$ $\left.1-(-1)^{c_{1}}\right\}$

$=2^{2 k}(-1)^{\operatorname{Tr}_{1}^{4 k}\left(\lambda w^{2^{k}+1}\right)}$.

Therefore, $f(x)$ is a bent function.

If $c_{2}=1$, then from (2.24) and (2.26), we have

$\widehat{\chi_{f}}(w)=\Delta_{1}+\Delta_{2}=\frac{1}{2} 2^{2 k}(-1)^{T r_{1}^{4 k}\left(\lambda w^{2^{k}+1}\right)}\left\{1+(-1)^{c_{1}}-\right.$ $\left.1+(-1)^{c_{1}}\right\}$

$=2^{2 k}(-1)^{T r_{1}^{4 k}\left(\lambda w^{2^{k}+1}\right)}\left\{(-1)^{c_{1}}\right\}$

$=\left\{\begin{array}{ll}2^{2 k}(-1)^{T r_{1}^{4 k}\left(\lambda w^{2^{k}+1}\right)} ; & c_{1}=0 \\ -2^{2 k}(-1)^{T r_{1}^{4 k}\left(\lambda w^{2^{k}+1}\right)} ; & c_{1}=1\end{array}\right.$.

Therefore, $f(x)$ is a bent function.

Let us suppose that $t_{1}=1, t_{2}=t_{3}=0$, then

$$
\Delta_{1}=\frac{1}{2} 2^{2 k}(-1)^{T r_{1}^{4 k}\left(\lambda w^{2^{k}+1}\right)}\left\{1+(-1)^{c_{1}}\right\}
$$

and

$$
\begin{aligned}
& \Delta_{2}=\frac{1}{2} 2^{2 k}(-1)^{\operatorname{Tr}_{1}^{4 k}\left(\lambda w^{2^{k}+1}\right)+c_{2}}\left\{1-(-1)^{c_{1}+1}\right\} \\
& =\frac{1}{2} 2^{2 k}(-1)^{T r_{1}^{4 k}\left(\lambda w^{2^{k}+1}\right)+c_{2}}\left\{1+(-1)^{c_{1}}\right\} .
\end{aligned}
$$

Therefore,

$$
\begin{gathered}
\left.\widehat{\chi_{f}}(w)=\Delta_{1}+\Delta_{2}=\frac{1}{2} 2^{2 k}(-1)^{\operatorname{Tr}_{1}^{4 k}\left(\lambda w^{2^{k}+1}\right.}\right)\left\{1+(-1)^{c_{1}}+\right. \\
\left.(-1)^{c_{2}}+(-1)^{c_{1}+c_{2}}\right\} .
\end{gathered}
$$

If $c_{2}=0$, then (2.27) becomes

$$
\begin{aligned}
& \widehat{\chi_{f}}(w)=\Delta_{1}+\Delta_{2}=\frac{1}{2} 2^{2 k}(-1)^{T r_{1}^{4 k}\left(\lambda w^{2^{k}+1}\right)}\left\{1+(-1)^{c_{1}}+\right. \\
& \left.1+(-1)^{c_{1}}\right\} \\
& =2^{2 k}(-1)^{T r_{1}^{4 k}\left(\lambda w^{2^{k}+1}\right)}\left\{1+(-1)^{c_{1}}\right\} \\
& =\left\{\begin{array}{cc}
2^{2 k+1}(-1)^{T r_{1}^{4 k}\left(\lambda w^{2^{k}+1}\right)} ; & c_{1}=0 . \\
0 ; & c_{1}=1
\end{array}\right.
\end{aligned}
$$

If $c_{2}=1$, then (2.27) becomes

$$
\begin{aligned}
& \widehat{\chi_{f}}(w)=\Delta_{1}+\Delta_{2}=\left.\frac{1}{2} 2^{2 k}(-1)^{\operatorname{Tr}_{1}^{4 k}\left(\lambda w^{2^{k}+1}\right.}\right)\left\{1+(-1)^{c_{1}}-\right. \\
&\left.1+(-1)^{c_{1}+1}\right\}
\end{aligned}
$$




$$
\begin{aligned}
& =\frac{1}{2} 2^{2 k}(-1)^{\operatorname{Tr}_{1}^{4 k}\left(\lambda w^{2^{k}+1}\right)}\left\{1+(-1)^{c_{1}}-1-(-1)^{c_{1}}\right\} \\
& =0 .
\end{aligned}
$$

From (2.28) and (2.29), it is clear that $f(x)$ is semi-bent and its Walsh spectrum is $\left\{0, \pm 2^{2 k+1}\right\}$ when $t_{1}=1, t_{2}=t_{3}=0$. In a similar manner, we can prove that $f(x)$ is semi-bent when either $t_{1}=t_{2}=0, t_{3}=1$ or $t_{1}=t_{3}=0, t_{2}=1$.

Example 2.3 Let $k=2$ and $\zeta$ be the primitive element of $F_{2^{8}}$ generated by the primitive polyomial $x^{8}+x^{4}+x^{3}+x^{2}+1$. If $\lambda=\zeta^{34}, a=\zeta^{248}, b=\zeta^{15}$ and $c=\zeta^{143}$, then the function $f(x)$ defined as

$$
\begin{gathered}
f(x)=\operatorname{Tr}_{1}^{8}\left(\zeta^{34} x^{5}\right)+\operatorname{Tr}_{1}^{8}\left(\zeta^{248} x\right) \operatorname{Tr}_{1}^{8}\left(\zeta^{15} x\right)+ \\
\operatorname{Tr}_{1}^{8}\left(\zeta^{248} x\right) \operatorname{Tr}_{1}^{8}\left(\zeta^{143} x\right)
\end{gathered}
$$

is a bent function. If we take $a=\zeta^{248}, b=\zeta^{15}$ and $c=\zeta^{238}$, then we have $\operatorname{Tr}_{1}^{8}\left(\lambda\left(a^{2^{k}} b+a b^{2^{k}}\right)\right)=0, \operatorname{Tr}_{1}^{8}\left(\lambda\left(a^{2^{k}} c+\right.\right.$ $a c 2 k)=0$ and $\operatorname{Tr} 18(\lambda b 2 k c+b c 2 k)=1$. So, the function $f(x)$ as defined in (2.30) is a semi-bent function.

\section{ACKNOWLEDGMENTS}

Authors acknowledge the support of UGC-SAP.

\section{REFERENCES}

[1] Rothaus, O. 1976. On bent functions. J. Combinat. Theory, Ser. A. Vol. 20, No. 3, pp. 300-305.

[2] Kumar, P. V., Scholtz, R. A. and Welch, L. R. 1985. Generalized bent functions and their properties. J. Comb. Theory(A). 40, pp. 90-107.

[3] Carlet, C. 2010. Boolean functions for cryptography and error correcting codes. Boolean Models and Methods in Mathematics, Computer Science and Engineering. Vol. 2, pp. 257.

[4] MacWilliams, F. J. and Sloane, N. J. A. 1977. The theory of error correcting codes. Vol. 16, Elsevier.

[5] Olsen, J. R., Scholtz, A. and Welch, L. 1982. Bent function sequences. IEEE Trans. Inf Theory. Vol. 28, No. 6, pp. 858-864.

[6] Tan, Y., Pott, A. and Feng, T. 2010. Strongly regular graphs associated with ternary bent functions. J. Combin. Theory Ser A. Vol. 117, No. 6, pp. 668-682.

[7] Chee, Y. M., Tan, Y. and Zhang, X. De. 2011. Strongly regular graphs constructed from p-ary bent functions. J. Algebr. Comb. Vol. 34, No. 2, pp. 251-266.

[8] Sharma, P. L. and Kumar, S. 2014. Balanced Incomplete Block Design (BIBD)Using Hadamard Rhotrices, International Journal of Technology, Vol. 4, No. 1, P. 6266.
[9] Sharma, P. L. and Kumar, S. 2014. Some applications of Hadamard rhotrices to design balanced incomplete block, International J. of Math. Sci. \& Engg. Appls. Vol. 8, No. II, P. 389-404.

[10] Sharma, P. L., Kumar, S. and Rehan, M. 2013. On Hadamard Rhotrix over Finite Field, Bulletin of Pure and Applied Sciences, Vol. 32 E (Math \& Stat.), No.2, P. 181-190.

[11] Sharma, P. L. and Kumar, S. 2013. On Construction of MDS Rhotrices from Companion Rhotrices over Finite Field. International Journal of Mathematical Sciences, Vol.12, No. 3-4, P. 271-286.

[12] Sharma, P. L., Sharma, Shabnam and Dhiman, Neetu 2014. Construction of infinite sequences of irreducible polynomials using Kloosterman sums, Bulletin of Pure and Applied Sciences, Vol. 33 E (Math. \& Stat.), No. 2, P. 161-168.

[13] Canteaut, A., Charpin, P. and Kyureghyan, G. 2008. A new class of monomial bent functions Finite Fields and Their Appl. Vol. 14, No. 1, pp. 221-241.

[14] Khoo, K., Gong, G. and Stinson, D. R. 2006. A new characterization of semi-bent and bent functions on finite fields. Des. Codes Cryptogr. Vol. 38, No. 2, pp. 279295.

[15] Charpin, P., Pasalic, E. and Tavernier, C. 2005. On bent and semi-bent quadratic Boolean functions. IEEE Trans. Inf. Theory. Vol. 51, No. 12, pp. 4286-4298.

[16] Helleseth, T. and Kholosha, A. 2010. New binomial bent functions over the finite fields of odd characteristic. IEEE.Trans. Inf. Theory. Vol. 56, No. 9, pp. 4646-4652.

[17] Leander, G. and Kholosha, A. 2006. Bent functions with $2^{r}$ Niho exponents. IEEE Trans. Inf. Theory. Vol. 52, No. 12, pp. 5529-553.

[18] Mesnager, S. 2015. Bent functions from spreads. Contemporary mathematics. Vol. 632, pp. 295-316.

[19] Sharma, P. L. and Dhiman, Neetu. 2016. Generalization of cross-correlation in Boolean functions and some generalized constructions in gbent functions. AsianEuropean Journal of Mathematics. Vol. 9, No. 1 (1650019).

[20] Sharma, P. L. and Dhiman, Neetu. 2016. A characterization theorem for gbent function over $Z_{16}$. Journal of Combin. Inf. and System Sciences. Vol. 41, Nos. 1-2, pp. 47-55.

[21] Xu, Guangkui, Cao, Xiwang and Xu, Shangding. 2015. Several new classes of Boolean functions with few Walsh transform values. arXiv: 1506.04886v 1 [cs.IT]. 